\title{
BMJ Open Comparative evaluation of novel screening strategies for colorectal cancer screening in China (TARGET-C): a study protocol for a multicentre randomised controlled trial
}

\author{
Hongda Chen, ${ }^{1} \mathrm{Ni} \mathrm{Li}^{1}{ }^{1}$ Jufang Shi, ${ }^{1}$ Jiansong Ren, ${ }^{1}$ Chengcheng Liu, ${ }^{1}$ \\ Yueming Zhang, ${ }^{2}$ Zheng Jiang, ${ }^{3}$ Zhihui Zhang, ${ }^{4}$ Min Dai ${ }^{1}$
}

To cite: Chen H, Li N, Shi J, et al. Comparative evaluation of novel screening strategies for colorectal cancer screening in China (TARGET-C): a study protocol for a multicentre randomised controlled trial. BMJ Open 2019;9:e025935. doi:10.1136/ bmjopen-2018-025935

\section{- Prepublication history for} this paper is available online. To view these files, please visit the journal online (http://dx.doi. org/10.1136/bmjopen-2018025935).

$\mathrm{HC}$ and NL contributed equally.

Received 9 August 2018

Revised 25 February 2019

Accepted 1 March 2019
Check for updates

(C) Author(s) (or their employer(s)) 2019. Re-use permitted under CC BY-NC. No commercial re-use. See rights and permissions. Published by BMJ.

For numbered affiliations see end of article.

Correspondence to

Min Dai;

daimin2002@hotmail.com

\section{ABSTRACT}

Introduction Screening for colorectal cancer (CRC) is effective in reducing the disease burden. However, highlevel evidence from randomised controlled trials on the effectiveness of CRC screening modalities is still lacking. We will conduct a large-scale multicentre randomised controlled trial in China to evaluate the effectiveness and cost-effectiveness of different CRC screening strategies. Methods and analysis 20000 eligible participants aged 5074 years are enrolled in five provinces in China. After providing signed informed consent, the participants will be randomised into one of the three screening groups: (1) one-time colonoscopy ( $n=4000)$, (2) annual faecal immunochemical test ( $F I T)(n=8000)$ and (3) annual risk-adapted screening strategy $(n=8000)$. The risk-adapted screening strategy will use an established CRC risk scoring system, the Asia-Pacific Colorectal Screening score. Participants at high risk of CRC will be referred for colonoscopy, while participants at low risk will be referred for an FIT. Information on clinical reports, epidemiological risk factors and health economic factors will be collected and stored in a web-based data management system. We will further request the participants to donate blood, faecal and saliva samples before conducting the colonoscopy. The primary outcome will be the detection rate of advanced colorectal neoplasia and the secondary outcomes will include the rates of CRC-related mortality, incidence of CRC, participation and complications. The study will last for at least 4 years and the cohort will be followed for 10 years to adequately answer the scientific questions.

Ethics and dissemination This study was approved by the Ethics Committee of the National Cancer Center/Cancer Hospital, the Chinese Academy of Medical Sciences and Peking Union Medical College (18-013/1615). The results of the study will be submitted for publication in peer-reviewed journals and will be discussed by policy and decision makers. Trial registration number ChiCTR1800015506.

\section{INTRODUCTION}

Colorectal cancer (CRC) is the third most commonly diagnosed cancer and the second most common cause of cancer-related death worldwide. ${ }^{1}$ In China, with estimated 376300

\section{Strengths and limitations of this study}

- This is the first large-scale population-based trial in China to compare the effectiveness and cost-effectiveness of three different colorectal cancer (CRC) screening strategies targeting adults aged 50-74 years.

- A comprehensive health-economic evaluation will be performed to evaluate the cost-effectiveness of the different screening arms and policy advice will, therefore, be provided based on the study findings.

- Prospective biospecimens collected before screening colonoscopy will be a valuable resource to explore novel biomarkers for the early detection of CRC in further research.

- The sample sizes of the study population may not be adequate to compare mortality reduction among the three screening arms after long-term follow-up.

newly diagnosed CRC cases and 191000 CRC-related deaths in 2015, the incidence and mortality ranked fourth and fifth of all cancer types, respectively. The incidence and mortality of CRC in China have been steadily increasing in recent decades. ${ }^{2}$ Therefore, the establishment of strategies to curb the rising momentum of CRC in China is strongly required.

Evidence from randomised controlled trials and observational studies has demonstrated that screening could reduce the burden of CRC. $^{3-5}$ The established screening modalities include colonoscopy, flexible sigmoidoscopy and stool-based tests such as the faecal occult blood test (FOBT), which have been widely used in many screening programmes worldwide. $^{6-8}$ Colonoscopy is the gold standard for CRC. However, in population-based screening programmes, colonoscopy is limited by low compliance rates, potential complications, 
high costs and limited resources. ${ }^{9}{ }^{10}$ Guaiac-based FOBT (gFOBT) was introduced in the 1980s. Although the sensitivity of gFOBT for detecting CRC is not optimal, randomised controlled trials demonstrated that screening by gFOBT yielded a reduction in CRC mortality. ${ }^{5}$ The newly developed faecal immunochemical test (FIT) for haemoglobin showed superior diagnostic performance compared with that of traditional gFOBT. ${ }^{11}$ However, evidence from randomised controlled trials evaluating the screening efficacy of FIT is still lacking, especially in the Chinese population. ${ }^{11}$

Current guidelines recommend CRC screening for average-risk adults starting at 50 years of age ${ }^{12-15}$ However, in countries with unbalanced and limited healthcare resources, identification of high-risk populations and the development of risk-adapted screening strategies may be more cost-efficient than traditional screening strategies. Previous studies developed CRC risk scores based on environmental and/or genetic factors, which typically presented moderate diagnostic efficacy. ${ }^{16}$ The combination of risk scores and established screening modalities such as colonoscopy and FIT had been proposed and has shown promising diagnostic performance. ${ }^{131718}$ However, further validation of such risk-adapted screening strategies in large prospective cohorts and randomised controlled trial are sparse.

Identification of biomarkers for the early detection of CRC is a promising area of research. Different types of biomarkers, including blood proteins, blood DNA methylation, faecal DNA, faecal microbiota and oral microbiota, have been associated with CRC and could be targets for the early detection of CRC. ${ }^{19}$ The use of ongoing screening trials to construct a biobank will be both timesaving and economical and will also be an important platform for future biomarker identification and validation.

CRC screening in China has been implemented in several regions over the past decades. ${ }^{20} 21$ However, high-quality evidence for the recommendation of CRC screening in the Chinese population is still lacking and in high demand. ${ }^{20}$ Therefore, we plan to conduct a population-based, multicentre, randomised controlled trial comparing colonoscopy, FIT and a novel risk-adapted CRC screening strategy in the Chinese population, with the following aims: (1) to establish a large-scale CRC screening cohort with long-term follow-ups in China, (2) to evaluate the effectiveness and cost-effectiveness of different CRC screening strategies in the Chinese population and (3) to construct a large epidemiological and clinical database and a biobank for further studies.

\section{METHODS AND ANALYSIS}

\section{Study setting and design}

This prospective, multicentre, randomised controlled trial will compare multiple CRC screening strategies in China. Participants who meet the study inclusion and exclusion criteria will be recruited in five provinces in China. We aim to recruit at least 20000 eligible participants at baseline. After obtaining signed informed consent, eligible participants will be randomly allocated into one of the three CRC screening groups in a 1:2:2 ratio (figure 1 ). A 4-year screening phase (with 1 year baseline screening and 3 years of follow-up screening) will be conducted for all participants and a subsequent passive follow-up phase

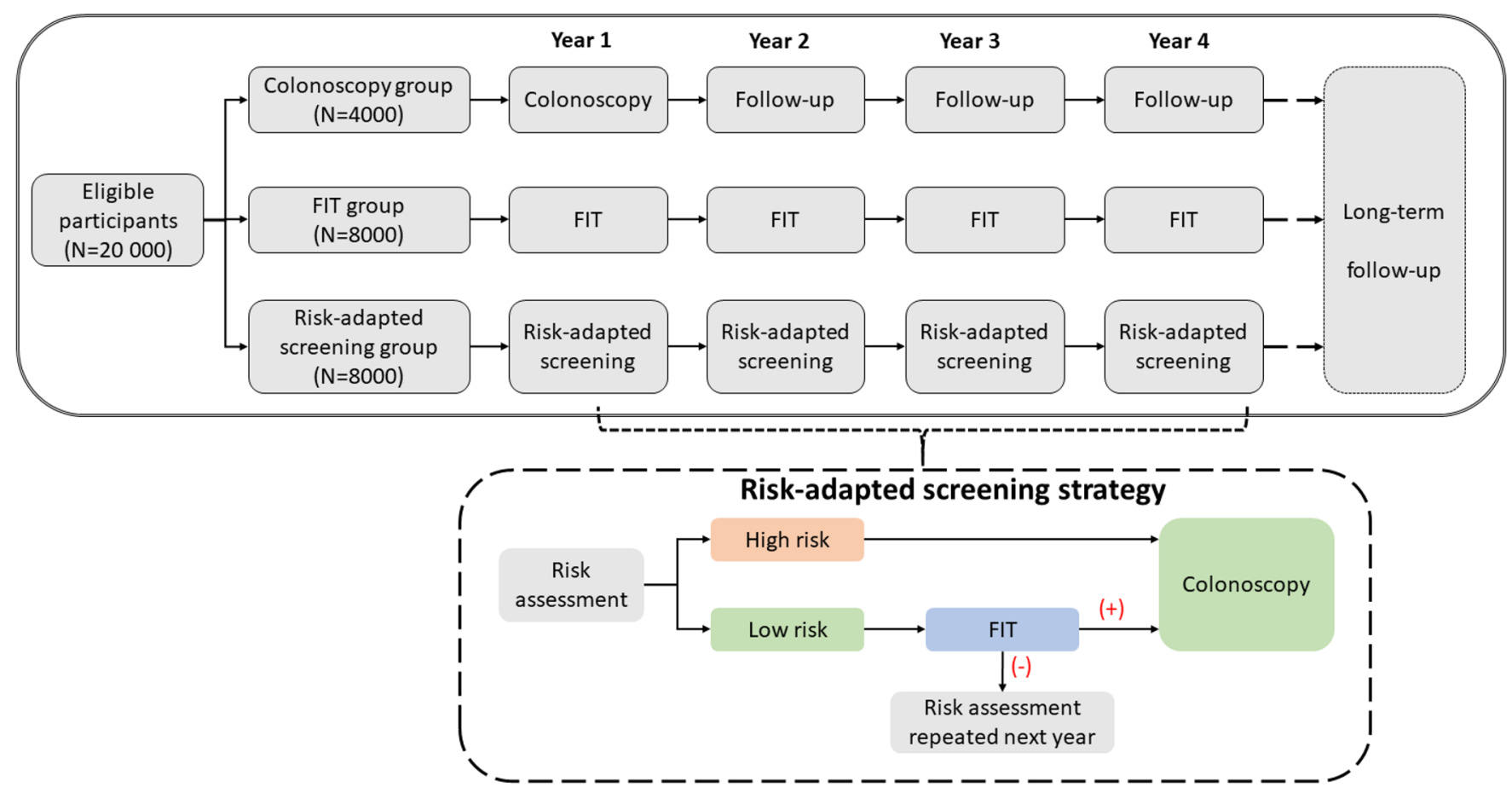

Figure 1 Standard Protocol Items: Recommendations for Interventional Trials flow diagram of the study design. FIT, faecal immunochemical test. 
will also be implemented until the scientific questions are answered adequately. Detailed information about the follow-up is described in the following section.

1. Colonoscopy group $(n=4000)$ : participants are recommended to undergo a one-time screening colonoscopy at baseline. Abnormal findings removed during colonoscopy will be sent to pathology for further analysis. In the following years, all participants will be interviewed to complete the follow-up questionnaire annually.

2. FIT group $(n=8000)$ : FITs are offered to the participants annually. Participants with positive FIT findings are recommended to undergo a diagnostic colonoscopy. Abnormal findings removed during colonoscopy will be sent to pathology for further analysis.

3. Risk assessment group $(n=8000)$ : CRC risk will be assessed using an established CRC risk stratification scoring system at baseline. For participants at high risk of CRC, screening colonoscopy will be offered. For participants at low risk of CRC, FITs will be offered and those with positive FIT results will be recommended to undergo further colonoscopy. During the annual follow-ups, participants with negative FIT results and those who have not had a screening colonoscopy will complete another round of risk assessment and the same screening procedures as at baseline will be offered. Participants who have already undergone screening colonoscopy will be provided no further screening intervention but the participants will complete a questionnaire annually during the study period.

\section{Randomisation and allocation procedure}

The randomisation will be conducted in a centralised, controlled manner. The leading institute (Cancer Hospital, Chinese Academy of Medical Sciences) is responsible for the generation of the randomisation scheme using a predefined seed from the statistical software R. Before recruitment, both the staff responsible for recruitment at each site and the participants will be blinded to the allocation results. The allocation results will be revealed after successful registration of the subject in a web-based data system. At the time of randomisation, each patient will be assigned a unique Study Identification Number (SIN), which will be used during the entire study period.

\section{Study population and recruitment}

Participants aged 50-74 years who live in the study region and are able to sign informed consent are eligible for this study. The exclusion criteria are (1) prior history of CRC; (2) prior history of colonic resection; (3) receipt of any kind of cancer-related therapy (except for non-melanoma skin cancer); (4) prior colonic examination, including colonoscopy, flexible sigmoidoscopy, CT colonography and barium enema within 5 years; (5) prior history of FOBT and faecal DNA test within 1year; (6) symptoms of lower gastrointestinal tract disease warranting colonoscopic evaluation, including (a) more than one episode of rectal bleeding within the past 6 months, (b) documented iron deficiency anaemia and (c) significant documented unintentional weight loss ( $>10 \%$ of baseline weight) over 6 months; and (7) significant comorbidity that would preclude benefit from screening or pose a significant risk to the performance of colonoscopy (eg, severe lung disease, end-stage renal disease, end-stage liver disease, severe heart failure or recent diagnosis of cancer, with the exception of non-melanoma skin cancer).

The recruitment procedures will include the following steps:

1. Recruitment of potential participants aged $50-74$ years in the selected communities and checking for eligibility by trained study staff.

2. Signed written informed consent obtained from the eligible participants by trained study staff.

3. Registration of the participant in the web-based data management system, SIN assignment and randomisation results revealed.

4. Conducting respective intervention strategies per protocol.

\section{Interventions}

Colonoscopy

Standard clinical procedures for the screening colonoscopy will be followed, including appointment; obtaining informed consent; routine blood testing for infectious diseases including hepatitis B virus, hepatitis $\mathrm{C}$ virus and HIV infections (if required by the hospitals, otherwise not implemented); distribution of bowel preparation drugs; diet control; anaesthesia (if required by the participants); and colonoscopy examination. The colonoscopies will be performed by experienced endoscopists with $>5$ years of experience in performing colonoscopy. Abnormal findings during colonoscopy will be carefully checked under standard clinical procedures and tissue specimens will be collected for further pathology diagnosis. Any findings during colonoscopy are required to be documented photographically. Clinical information such as the examination duration, sedation status, completeness of colonoscopy, bowel preparation status, complications, polyp features (number, position, size, colour and shape), description of other abnormal findings, as well as pathology diagnosis will be collected and documented in the web-based data management system.

For quality control, an expert panel will be formed, including experienced endoscopists and pathologists. Each year, a selection of colonoscopy and pathology documentation will be assessed by the expert panel and review reports will be transferred to the respective physicians regarding their performance.

\section{Faecal immunochemical test}

FITs for human haemoglobin will be provided by the study staff to participants after successful registration in this study. The FIT used in this study is a self-administered qualitative test, providing an endpoint, that is, visually interpreted as positive or negative by eye if the faecal haemoglobin concentration exceeds the 
Table 1 Risk factors and respective proposed points for Asia-Pacific Colorectal Screening scores to be used in this trial

\begin{tabular}{lll}
\hline Risk factor & Criteria & Points \\
\hline Age (years) & $<50$ & 0 \\
& $50-69$ & 1 \\
& $\geq 70$ & 2 \\
Sex & Female & 0 \\
& Male & 1 \\
Family history of colorectal & Absent & 0 \\
cancer in a first-degree & Present & 1 \\
relative & No & 0 \\
Smoking & Current or past & 1 \\
& $<23$ & 0 \\
BMl & $\geq 23$ & 1 \\
\hline
\end{tabular}

BMI calculated as weight $(\mathrm{kg}) /$ height $^{2}\left(\mathrm{~m}^{2}\right)$.

BMI, body mass index.

manufacturer-specific threshold $(100 \mathrm{ng} \mathrm{Hb} / \mathrm{mL}$ buffer, corresponding to $10 \mu \mathrm{g} \mathrm{Hb} / \mathrm{g}$ faeces). A previous pilot analysis demonstrated that the sensitivities of $76 \%$ and $37 \%$, respectively, for the detection of CRC and advanced adenomas, at a specificity of $92 \%$ (data not publicly available). The participants can submit the results to the study website along with the picture of the test window or will be interviewed by the study staff regarding the test results within 3 days of distributing the FIT. For participants with invalid test results, new test devices will be provided until a result is available. Participants with confirmed positive FIT results will be contacted and a follow-up colonoscopy will be arranged.

\section{CRC risk assessment}

This study will use an established CRC risk scoring system, the Asia-Pacific Colorectal Screening (APCS) score. ${ }^{22} 23$ The APCS score is derived from five common risk factors of CRC, including age, sex, family history of CRC in a first-degree relative, smoking and body mass index (BMI). In a previous study conducted in Hong Kong, the sensitivity, specificity, positive predictive value and negative predictive value of the risk score for detecting advanced neoplasms were $33.3 \%, 81.0 \%, 5.17 \%$ and $97.5 \%$, respectively, defining a score $\geq 4$ as high risk for CRC. ${ }^{23}$ Based on previous evidence, we designed the risk score system and detailed information shown in table 1. Generally, the participants of the risk-adapted screening group will be asked to complete a questionnaire including the above-mentioned risk factors. Participants with a score $\geq 4$ are defined to be at high risk of CRC, while those with a score $<4$ are defined to be at low risk of CRC. Participants will be informed about their evaluation results and receive the respective screening intervention as per the study protocol.

\section{Patient and public involvement}

During the process of recruitment, study staff will inform the participants about the research question, study design and screening intervention. The participants can quit the study and withdraw their informed consent at any time based on their priorities, experiences or preferences. The participants and the public had no role in the study design, recruitment and conduct. All screening interventions will be provided to the participants at no cost (compensated by this study), except for the subsequent therapeutic costs which must be paid by the participants themselves. At the recruitment phase, the study staff will inform the participants of the burden of the intervention and potential subsequent therapeutic procedure. The study staff will also disseminate to the participants a report summarising the screening results.

\section{Biospecimen collection and handling}

Participants who require colonoscopy will be invited to donate stool, saliva and blood samples prior to colonoscopy. Standard operating procedures (SOPs) regarding biospecimen collection, handling and storage have been formulated and will be followed.

For stool samples, collection devices (sample collection vials, ice bags, isothermal bags and operation brochures) will be distributed. On the day before the colonoscopy, the participants will be suggested to collect raw stool samples before taking bowel cleaning drugs for colonoscopy. The participants will be recommended to store the samples in the freezer or in the isothermal bags with ice bags until transported to the hospital. The samples will be stored at $-80^{\circ} \mathrm{C}$ immediately on receipt for future use.

For saliva samples, participants will be provided with sample collection tubes containing oral DNA stabilisation buffer during their visit to the hospital before the colonoscopy. Study staff will guide the participants on the saliva sample collection procedure. The collected samples will be aliquoted immediately and stored at $-80^{\circ} \mathrm{C}$ for future use.

Approximately $10 \mathrm{~mL}$ venous blood samples (including $5 \mathrm{~mL}$ ethylenediaminetetraacetic acid anticoagulated blood and $5 \mathrm{~mL}$ non-anticoagulated blood) will be drawn from the participants during their visit to the hospital before colonoscopy. Under the SOPs, the blood samples are to be centrifuged, aliquoted and stored at $-80^{\circ} \mathrm{C}$ for future use.

\section{Follow-up}

The study will conduct both active and passive follow-up. For the active follow-up, all the participants will be interviewed by trained study staff by telephone call, home visit or other contact methods for the collection of information such as physical examination, health status and outcome. For the passive follow-up, linkage data from the cancer registry system, death surveillance system and medical insurance and claim databases will be used to track the outcome of the participants. 


\section{Contamination evaluation}

During the study period, the study team will contact the participants to evaluate the status of CRC beyond the study protocol. The extra screening examinations attended by the participants during the study period are not allocated by randomisation and, therefore, may introduce bias to the study results. To evaluate the contamination status of this study, all participants whose screening findings are negative will complete one round of questionnaire interview in the fourth year of the study. Information regarding the history of diagnostic or colonic examination screening will be collected and assessed. We anticipate controlling the contamination rate to be $<10 \%$. The final analysis report will consider the contamination when estimating the effects of screening.

\section{Outcome measures}

The primary outcome is the detection rate of advanced colorectal neoplasia (CRC and advanced adenoma). The secondary outcomes include the rates of CRC mortality, detection of any neoplasm, compliance and complications.

\section{Data collection \\ Epidemiological risk factor investigation}

A standardised epidemiological questionnaire will be administered by trained interviewers to all participants to investigate the risk factors of CRC. Information including sociodemographic factors, history of bowel disease and clinical treatment, living habits, disease history and family history of cancer will be collected and stored in a web-based data management system.

\section{Health economic information}

A comprehensive health economic evaluation will be conducted. Questionnaires including the EuroQol five dimensions questionnaire (EQ-5D) and EQ-5D-5L will be used to measure the health state of the participants. The direct costs of materials, equipment, personnel, drug and other resources will be collected from all participating sites to estimate the cost-effectiveness of different screening strategies in this clinical trial.

\section{Data monitoring committee}

A data monitoring committee comprising epidemiologists, endoscopists, pathologists and colorectal surgeons will monitor data collection and analyses. All data will be transmitted to the Central Data Management Team at the National Cancer Center of China/Cancer Hospital Chinese Academy of Medical Sciences, where the databases are constructed and analyses are performed. In addition, any adverse events, such as perforation and bleeding, will be recorded in standardised forms by the study sites and will also be reported to the ethics committee for their records.

\section{Statistical considerations}

\section{Sample sizes}

Sample sizes were calculated based on the evaluation of primary outcomes; that is, the detection rate of advanced colorectal neoplasia. The hypothesis was that this rate in the risk-adapted screening group was superior to that of the FIT group and non-inferior to that of the colonoscopy group. According to previous studies, the reference advanced neoplasia detection rates of colonoscopy, FIT and risk-adapted screening groups were $6.5 \%, 1.8 \%$ and $5.0 \%$, respectively. ${ }^{13}$ We assumed compliance rates of $50 \%-70 \%$ for colonoscopy, $60 \%-90 \%$ for FIT and $60 \%-90 \%$ for the risk-adapted screening strategy and an overall loss-to-follow-up rate of $10 \%$. For the comparison between the risk-adapted screening strategy and the FIT groups for different scenarios of compliance rates, the largest sample size needed was 6550 at a significance level of $\alpha=0.05$, power of 0.8 and superiority margin $(\delta)$ of -0.005 . For comparison between the risk-adapted screening and colonoscopy groups, assuming respective compliance rates of $85 \%$ and $60 \%$, the required sample sizes were 6032 and 3016, respectively, for a significance level of $\alpha=0.05$, power of 0.8 and non-inferiority margin $(\delta)$ of -0.001 . Therefore, the sample sizes in this study design (4000 for the colonoscopy group, 8000 for the FIT group and 8000 for the risk-adapted screening group) will accomplish the study hypotheses.

\section{Statistical analyses}

The primary outcome analysis will be comparisons of histologically confirmed CRC and advanced adenoma between the three intervention arms, taking the compliance rate into consideration. Intention-to-treat and per-protocol analyses will be conducted. For secondary outcomes, the mortality rate will be calculated as the ratio of the number of deaths due to CRC to the person-years at risk for each group. Person-years will be estimated from the time of randomisation to the diagnosis date of CRC, death or censoring at the end of the study. The incidence rate will be estimated similarly. Chi-square and t-tests will be used to compare categorical and continuous variables between the two groups, respectively. The Cox proportional hazards regression model will be adopted to examine the differences in incidence and mortality between different screening groups. For health economic evaluations, Markov models will be developed to evaluate the cost-effectiveness of different CRC screening strategies in China. Statistical software such as SAS V.9.2, R V.3.4.1 (R Foundation for Statistical Computing, Vienna, Austria) and TreeAge Pro 2016 (TreeAge Software, Williamstow, Massachusetts, USA) will be used for the data analyses.

\section{Ethics and dissemination}

The results of the study will be submitted for publication to peer-reviewed journals and conferences following the Consolidated Standards of Reporting Trials guidelines. The results will be discussed by policy and decision 
makers. Access to the detailed research plan, participant-level dataset and code for statistical analysis will be granted based on reasonable requests after the publication of the study.

\section{Trial status}

This screening trial is currently in the participant enrolment phase. A total of 1600 eligible participants have been randomised and are under respective CRC screening as of August 2018. We anticipate the full analysis to be finalised in December 2021.

\section{DISCUSSION}

Our study aims to evaluate the effectiveness and cost-effectiveness of three CRC screening strategies in China. To our knowledge, this is the first large-scale randomised controlled trial on CRC screening based on a community population in China. Colonoscopy is the gold standard for CRC screening and FIT is the most widely used non-invasive CRC screening test. However, the magnitude of the effect of colonoscopy and FIT in population-based CRC screening is uncertain due to a lack of evidence from randomised controlled trials. To date, three large-scale randomised controlled trials (SCREESCO, CONFIRM and COLONPREV) have compared colonoscopy or FIT screening regarding CRC incidence and mortality. ${ }^{25-28}$ All three trials are ongoing and being conducted in Europe and North America. Our study will be the first large-scale CRC screening trial in Asia. In addition, we also include a novel risk-adapted screening strategy that incorporates risk assessment with established screening methods. Our study will provide strong evidence on the effectiveness and feasibility of different strategies for CRC screening in China.

In recent years, the burden of CRC has been increasing in East-Asia due to changes in diet and Westernised lifestyles. ${ }^{29}$ Countries including China, Japan and South Korea have implemented organised screening programmes. For instance, in Japan, the CRC screening programme initiated in 1992 uses FIT as the main screening method, the cost of which is covered by the national health insurance. ${ }^{30}$ In China, individuals aged 40-74 years are screened with FOBT or colonoscopy based on clinical risk indexes in some regions but not the entire country. ${ }^{20}$ Furthermore, the most appropriate techniques for different populations in China are still under debate. The results of our study will, therefore, provide high-level evidence to design CRC screening strategies for China and will also provide an essential reference for other countries.

We plan to finish the baseline recruitment and baseline screening for this study before June 2019 and will have a total of three rounds of screening intervention FIT and risk-adapted screening groups. Long-term passive follow-up will also be conducted to determine the health outcomes of the participants for the evaluation of the long-term effect of CRC screening. Our study has several strengths. First, the prospective randomised design will minimise selection bias and provide high-level evidence compared with those of other designs such as cross-sectional studies. In addition, except for active follow-up, we will also implement passive follow-up using multiple resources such as cancer registry, death surveillance system and medical insurance and claim databases to track the outcomes of the study participants. We will also construct a large biobank using prospectively collected specimens. This biobank will serve as an essential platform for biomarker identification and validation for further investigations.

The major challenges of this study are control of loss to follow-up and quality control of a multicentre project. To address such concerns, we will employ experienced study staff to regularly contact and visit the participants. Moreover, a health education campaign will be conducted to improve health literacy by means of lectures, videos, advertisements and social media. For quality control, we will build an expert panel including experts in epidemiology, endoscopy, pathology and surgery. A capacity training workshop will be held annually and a selection of study reports will be reviewed to ensure study quality.

In summary, this large-scale multicentre randomised controlled trial will compare three CRC screening strategies. Successful implementation of this study will provide strong evidence on the effectiveness and cost-effectiveness of CRC screening and provide an essential reference for policy-makers to design national screening programmes.

\section{Author affiliations}

${ }^{1}$ Office of Cancer Screening, National Cancer Center/National Clinical Research Center for Cancer/Cancer Hospital, Chinese Academy of Medical Sciences and Peking Union Medical College, Beijing, China

${ }^{2}$ Department of Endoscopy, National Cancer Center/National Clinical Research Center for Cancer/Cancer Hospital, Chinese Academy of Medical Sciences and Peking Union Medical College, Beijing, China

${ }^{3}$ Department of Colorectal Surgery, National Cancer Center/National Clinical Research Center for Cancer/Cancer Hospital, Chinese Academy of Medical Sciences and Peking Union Medical College, Beijing, China

${ }^{4}$ Department of Pathology, National Cancer Center/National Clinical Research Center for Cancer/Cancer Hospital, Chinese Academy of Medical Sciences and Peking Union Medical College, Beijing, China

Acknowledgements The authors thank the participating sites (Zhejiang Cancer Hospital, Anhui Provincial Cancer Hospital, Xuzhou Cancer Hospital, Hunan Cancer Hospital, and Yunnan Cancer Hospital), the Centers for Disease Control and Prevention of the participating cities and the community staff and participants for their contributions. We also thank Dr. Prudence Carr for the English proofreading of this manuscript.

Contributors $\mathrm{HC}, \mathrm{NL}$ and $\mathrm{MD}$ designed the study protocol. $\mathrm{HC}$ and $\mathrm{NL}$ drafted the manuscript. JS, JR, CL, YZ, ZJ, ZZ and MD critically reviewed and revised the manuscript. All authors read and approved the final manuscript.

Funding This work was supported by the CAMS Innovation Fund for Medical Sciences (2017-I2M-1-006) and the National Natural Science Foundation of China (81703309).

Competing interests None declared.

Patient consent for publication Not required.

Ethics approval This study was approved by Ethics Committee of the National Cancer Center/Cancer Hospital, the Chinese Academy of Medical Sciences and Peking Union Medical College (approved number: 18-013/1615) and the protocol was registered in the Chinese Clinical Trial Registry (registration number: ChiCTR1800015506). 
Provenance and peer review Not commissioned; externally peer reviewed.

Open access This is an open access article distributed in accordance with the Creative Commons Attribution Non Commercial (CC BY-NC 4.0) license, which permits others to distribute, remix, adapt, build upon this work non-commercially, and license their derivative works on different terms, provided the original work is properly cited, appropriate credit is given, any changes made indicated, and the use is non-commercial. See: http://creativecommons.org/licenses/by-nc/4.0/.

\section{REFERENCES}

1. Bray F, Ferlay J, Soerjomataram I, et al. GLOBOCAN estimates of incidence and mortality worldwide for 36 cancers in 185 countries. CA Cancer J Clin 2018;68:394-424.

2. Chen W, Zheng R, Baade PD, et al. Cancer statistics in China, 2015. CA Cancer J Clin 2016;66:115-32.

3. Zorzi M, Fedeli U, Schievano E, et al. Impact on colorectal cancer mortality of screening programmes based on the faecal immunochemical test. Gut 2015;64:784-90.

4. Brenner H, Stock C, Hoffmeister M. Effect of screening sigmoidoscopy and screening colonoscopy on colorectal cancer incidence and mortality: systematic review and meta-analysis of randomised controlled trials and observational studies. BMJ 2014;348:g2467.

5. Hewitson P, Glasziou P, Watson E, et al. Cochrane systematic review of colorectal cancer screening using the fecal occult blood test (hemoccult): an update. Am J Gastroenterol 2008;103:1541-9.

6. Brenner H, Kloor M, Pox CP. Colorectal cancer. Lancet 2014;383:1490-502.

7. Schreuders EH, Ruco A, Rabeneck L, et al. Colorectal cancer screening: a global overview of existing programmes. Gut 2015;64:1637-49.

8. Whitlock EP, Lin JS, Liles E, et al. Screening for colorectal cancer: a targeted, updated systematic review for the U.S. Preventive Services Task Force. Ann Intern Med 2008;149:638-58.

9. Senore C, Inadomi J, Segnan N, et al. Optimising colorectal cancer screening acceptance: a review. Gut 2015;64:1158-77.

10. Robertson DJ, Kaminski MF, Bretthauer M. Effectiveness, training and quality assurance of colonoscopy screening for colorectal cancer. Gut 2015;64:982-90.

11. Tinmouth J, Lansdorp-Vogelaar I, Allison JE. Faecal immunochemical tests versus guaiac faecal occult blood tests: what clinicians and colorectal cancer screening programme organisers need to know. Gut 2015;64:1327-37.

12. Lin JS, Piper MA, Perdue LA, et al. Screening for colorectal cancer: updated evidence report and systematic review for the US Preventive Services Task Force. JAMA 2016;315:2576-94.

13. Chiu HM, Ching JY, Wu KC, et al. A Risk-Scoring System Combined With a Fecal Immunochemical Test Is Effective in Screening High-
Risk Subjects for Early Colonoscopy to Detect Advanced Colorectal Neoplasms. Gastroenterology 2016;150:617-25.

14. Provenzale D, Jasperson K, Ahnen DJ, et al. National comprehensive cancer netwok. Colorectal Cancer Screening, Version 1.2015. J Natl Compr Canc Netw 2015;13:959-68. quiz 68.

15. Armaroli $P$, Villain $P$, Suonio $E$, et al. European Code against Cancer, 4th Edition: Cancer screening. Cancer Epidemiol 2015;39(Suppl 1):S139-52

16. Win AK, Macinnis RJ, Hopper JL, et al. Risk prediction models for colorectal cancer: a review. Cancer Epidemiol Biomarkers Prev 2012;21:398-410.

17. Jung YS, Park $\mathrm{CH}$, Kim NH, et al. A combination of clinical risk stratification and fecal immunochemical test is useful for identifying persons with high priority of early colonoscopy. Dig Liver Dis 2018:50:254-9.

18. Cubiella J, Digby J, Rodríguez-Alonso L, et al. The fecal hemoglobin concentration, age and sex test score: Development and external validation of a simple prediction tool for colorectal cancer detection in symptomatic patients. Int J Cancer 2017;140:2201-11.

19. Dickinson BT, Kisiel J, Ahlquist DA, et al. Molecular markers for colorectal cancer screening. Gut 2015;64:1485-94.

20. Goss PE, Strasser-Weippl K, Lee-Bychkovsky BL, et al. Challenges to effective cancer control in China, India, and Russia. Lancet Oncol 2014;15:489-538.

21. Guo C, Liu Q, Dai M. [Colorectal cancer screening: situation and prospect]. Zhonghua Yu Fang Yi Xue Za Zhi 2015;49:377-80.

22. Sung JJY, Wong MCS, Lam TYT, et al. A modified colorectal screening score for prediction of advanced neoplasia: A prospective study of 5744 subjects. J Gastroenterol Hepatol 2018;33:187-94.

23. Yeoh KG, Ho KY, Chiu HM, et al. The Asia-Pacific Colorectal Screening score: a validated tool that stratifies risk for colorectal advanced neoplasia in asymptomatic Asian subjects. Gut 2011;60:1236-41.

24. Imperiale TF, Ransohoff DF, Itzkowitz SH, et al. Multitarget stool DNA testing for colorectal-cancer screening. N Engl J Med 2014;370:1287-97.

25. Kaminski MF, Bretthauer M, Zauber AG, et al. The NordICC Study: rationale and design of a randomized trial on colonoscopy screening for colorectal cancer. Endoscopy 2012;44:695-702.

26. Quintero E, Castells A, Bujanda L, et al. Colonoscopy versus fecal immunochemical testing in colorectal-cancer screening. $N$ Engl J Med 2012;366:697-706.

27. CONFRIM trial. http://www.clinicaltrials.gov/ct2/show/NCT01239082 (Accessed 28 Apr 2018)

28. Screesco trial. http://www.clinicaltrials.gov/ct2/show/NCT02078804 (Accessed 28 Apr 2018).

29. Sung JJ, Lau JY, Goh KL, et al. Increasing incidence of colorectal cancer in Asia: implications for screening. Lancet Oncol 2005;6:871-6.

30. Saito $\mathrm{H}$. Colorectal cancer screening using immunochemical faecal occult blood testing in Japan. J Med Screen 2006;13(Suppl 1):S6-7. 